\title{
Reverse Geometry Hybrid Contact Lens Fitting in a Case of Donor-Host Misalignment after Keratoplasty
}

\author{
Rafael J Pérez-Cambrodí, Pedro Ruiz-Fortes, David P Piñero Llorens
}

\begin{abstract}
Purpose: To report the successful outcome obtained after fitting a new hybrid contact lens in a cornea with an area of donor-host misalignment and significant levels of irregular astigmatism after penetrating keratoplasty (PKP).
\end{abstract}

Materials and methods: A 41-year-old female with bilateral asymmetric keratoconus underwent PKP in her left eye due to the advanced status of the disease. One year after surgery, the patient referred a poor visual acuity and quality in this eye. The fitting of different types of rigid gas permeable contact lenses was performed, but with an unsuccessful outcome due to contact lens stability problems and uncomfortable wear. Scheimpflug imaging evaluation revealed that a donor-host misalignment was present at the nasal area. Contact lens fitting with a reverse geometry hybrid contact lens (Clearkone, SynergEyes Carlsbad) was then fitted. Visual, refractive, and ocular aberrometric outcomes were evaluated during a 1 -year period after the fitting.

Results: Uncorrected distance visual acuity improved from a prefitting value of $20 / 200$ to a best corrected postfitting value of $20 / 20$. Prefitting manifest refraction was +5.00 sphere and -5.50 cylinder at $75^{\circ}$, with a corrected distance visual acuity of 20/30. Higher order root mean square (RMS) for a $5 \mathrm{~mm}$ pupil changed from a prefitting value of $6.83 \mu \mathrm{m}$ to a postfitting value of $1.57 \mu \mathrm{m}$ ( $5 \mathrm{~mm}$ pupil). The contact lens wearing was referred as comfortable, with no anterior segment alterations.

Conclusion: The SynergEyes Clearkone contact lens seems to be another potentially useful option for the visual rehabilitation after PKP, especially in cases of donor-host misalignment.

Keywords: Hybrid contact lens, Keratoplasty, Keratoconus, Clearkone, Reverse geometry contact lens, Scheimpflug photography

How to cite this article: Pérez-Cambrodí RJ, Ruiz-Fortes $P$, Llorens DPP. Reverse Geometry Hybrid Contact Lens Fitting in a Case of Donor-Host Misalignment after Keratoplasty. Int J Kerat Ect Cor Dis 2013;2(2):69-72.

\section{Source of support: $\mathrm{Nil}$}

\section{Conflict of interest: None}

\section{INTRODUCTION}

Penetrating keratoplasty (PKP) or full-thickness corneal transplant is a surgical procedure which aims at restoring an adequate transparency and often allows correcting irregular astigmatism induced by a great variety of pathological conditions, including keratoconus. ${ }^{1,2}$ However, refraction after PKP is usually not predictable and in many cases the use of contact lenses (CLs) is needed to achieve adequate levels of visual acuity (VA) and visual quality. ${ }^{3}$ The main reason for a decreased VA after PKP is the presence of moderate to high levels of astigmatism, but also the induction of anisometropia or even the presence of an irregular corneal surface. ${ }^{4}$ All these conditions are indications for CLs fitting. ${ }^{5}$ The aim of the current case report is to show a successful visual rehabilitation achieved with the fitting of a new-generation hybrid contact lens of reverse geometry in an irregular cornea after PKP, with an area of donor-host misalignment.

\section{CASE REPORT}

A 41-year-old female attended to our clinic asking for a second opinion about the status of her eyes and the potential solution of her visual problems. She had been diagnosed of bilateral asymmetric keratoconus 10 years before. On clinical examination, refraction was +5 sphere $-6 \times 70^{\circ}$ cylinder in her right eye (RE) and +7 sphere $-12 \times 100^{\circ}$ cylinder in her left eye (LE). Best spectacle corrected visual acuity (BSCVA) was 20/25 in RE and 20/80 in LE. Keratometry surface regularity index (SRI) and surface asymmetry index (SAI) (TMS-1 Tomey) were in RE $6.9 \times$ $7.965^{\circ}, 0.92$ and 1.69 , respectively. In her LE keratometric data was $6.0 \times 8.4 \times 111^{\circ}$, SRI 2.08 and SAI 2.61. Anterior segment biomicroscopy (ASB) showed Vogt bilateral striae and significant central corneal thinning, especially in the LE. After the diagnosis of bilateral asymmetric keratoconus (grade II in RE and grade III-IV in LE), an aspheric RGP (rigid gas permeable) $\mathrm{CL}$ (base curve $7.50 \mathrm{~mm}$, total diameter $9.30 \mathrm{~mm}$, power +2 D) was fitted in the RE (Persecon E. Ciba Vision Corp, Duluth, GA, USA), achieving an acceptable physical fitting, comfortable wear and good BSCVA (20/20). In the LE, PKP was indicated and performed due to the advanced status of the disease.

One year after surgery, refraction was stable in LE: +5 sphere $-5.5 \times 95^{\circ}$ cylinder with a BSCVA of 20/30. The topographic exam (Pentacam, OCULUS Optikgeräte, $\mathrm{GmbH}$ Wetzlar, Germany) showed a moderate irregular corneal astigmatism (Fig. 1A). In addition, the Scheimpflug images showed a donor-host misalignment at the nasal area. Aspheric and reverse geometry RGP CLs fitting were performed with unsuccessful outcome due to the contact lens instability and reduced comfortable wearing time achieved. The fitting of a reverse geometry hybrid contact lens was then considered as a potentially useful option. Specifically, the SynergEyes Clearkone contact lens was fitted (SynergEyes, Carlsbad, CA, USA), a hybrid contact lens made of paflufocon D hem-iberfilcon A which is designed and commonly used for CL fitting in keratoconus. It should be considered that this modality of CL has a vault design with a reverse 
geometry landing system aimed at clearing the predominant irregularities of the keratoconic cornea. The RGP material of the center of the lens is a thermoset fluorosilicone acrylate copolymer, derived primarily from siloxane acrylate, trifluoroethyl methacrylate and methylmethacrylate with a water content of less than $1 \%$ (Paflufocon D) and a Dk (oxygen permeability) of 100 Fatt. The peripheral skirt material is a poly-hema hydrophilic copolymer (Iberfilcon A) with a water content of $27 \%$ and a Dk of 9.3 Fatt.

In our case, the parameters of the CL fitted were $300 \mu \mathrm{m}$ of vault and steep soft skirt, obtaining an optimal physical fitting pattern with an adequate push-up movement (Fig. 1B). The fluorogram obtained with this contact lens using high molecule fluorescein (FluoreSoft $0.35 \%$, Holles Laboratories Inc, Cohasset, MA, USA) showed a central corneal clearance with a bearing in the outer landing zone of the contact lens (Fig. 1C). A bubble located in the nasal peripheral area of the corneal graft was unavoidable due to the abrupt transition between the corneal graft edge and the host cornea induced by misalignment at this area (Fig. 1D). At 2 weeks after contact lens wearing began, the patient had a BCVA of 20/20 and an over-refraction of Plano. The patient referred a very significant improvement of LE visual quality which was consistent with the result of the postfitting aberrometric analysis obtained for a $5 \mathrm{~mm}$ pupil (Tracey Technologies Corp, Houston, Texas, USA) (Fig. 2). At 1 year of contact lens wear, the patient maintained a comfortable wearing, with the same level of visual quality and no alterations of the anterior segment.

\section{DISCUSSION}

Contact lens fitting in corneas after PKP is complex and a big challenge even for an experimented eye care practitioner. ${ }^{6}$ No general rules can be provided for all patients and different types of CLs have been demonstrated to be useful in such cases. ${ }^{7,8}$ Spherical and aspheric rigid gas permeable (RGP) ${ }^{4,9-12}$ CLs have been widely used after PKP but due to the frequent physical instability and consequent bad tolerance ${ }^{6}$ other designs have been tested as well, such as reverse geometry CLs. ${ }^{13-15}$ The size, alignment, shape and position of the donor graft in relation to the host tissue may determine the first lens to fit. Katsoulos et $\mathrm{al}^{13}$ reported some cases successfully fitted with reverse geometry hydrogel CLs after PKP. Specifically, a customized tricurve design was shown to better fit over a globular postoperative topographic pattern. ${ }^{13}$ However, if the graft is not aligned or is protrusive, as in the case presented here, hydrophilic CLs are not indicated because of the increased risk of adherences. Reverse geometry RGP lenses have shown to be efficacious, safe and predictable for the visual rehabilitation after PKP in oblate corneal profiles, providing high levels of visual acuity and comfortable tolerance. ${ }^{13-15}$ This option may be especially useful to avoid abrupt transitions between donor and host if a sufficient sagittal height is used.

Hybrid contact lenses are a good alternative when stability and comfort are difficult to achieve with other types of RGP lenses, a condition that can occur after PKP. Binder and Kopecky ${ }^{16}$ fitted the Softperm hybrid CL in 22 corneas after PKP, obtaining worse outcomes in those eyes

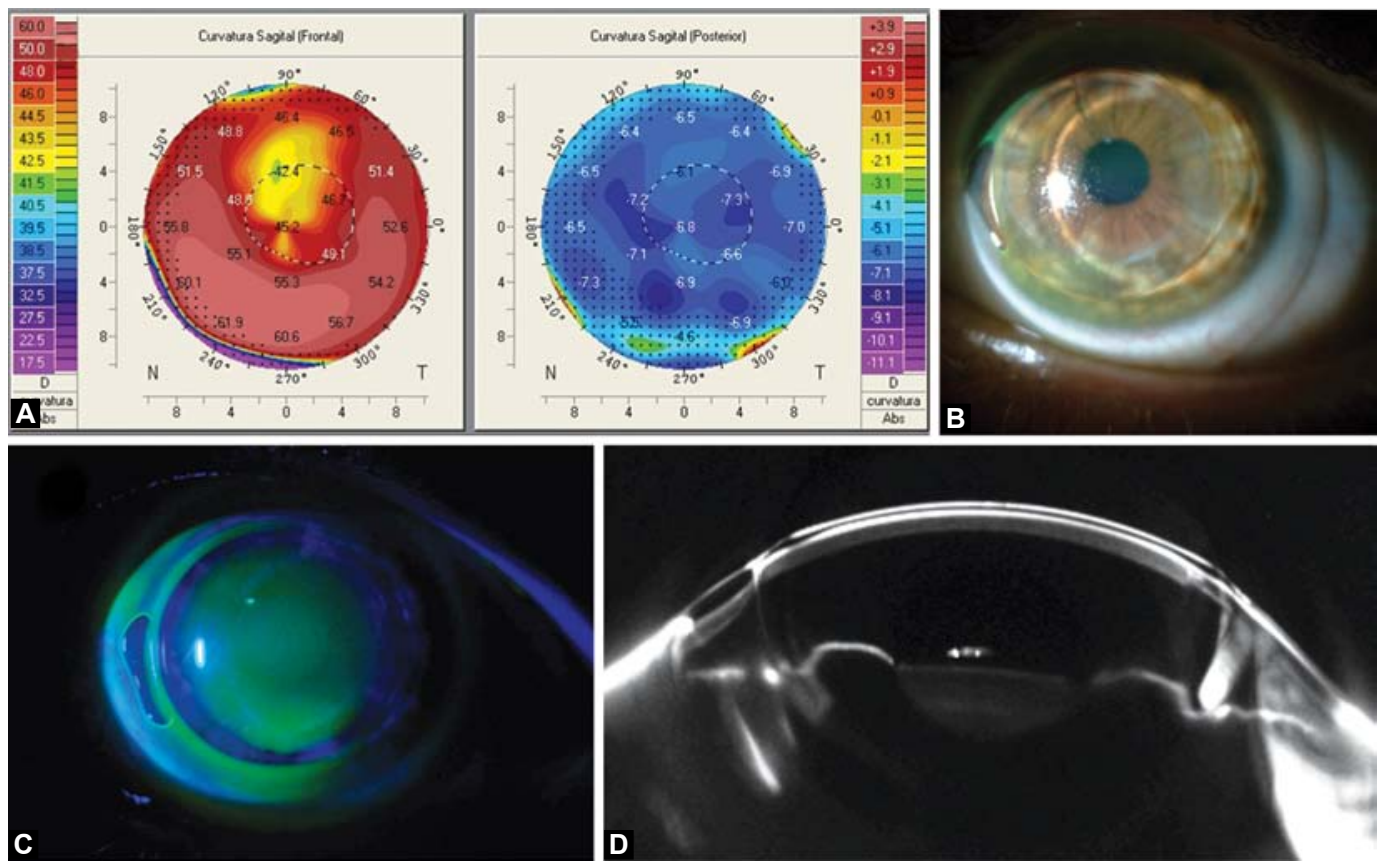

Figs 1A to D: Graphic summary of the case reported. (A) Anterior (left) and posterior (right) sagittal corneal topographic maps, (B) frontal image of the eye obtained with a camera adapted to a slit-lamp biomicroscopy, (C) fluorogram, (D) Scheimpflug image showing the relationship between the posterior surface of the contact lens and the anterior corneal surface 


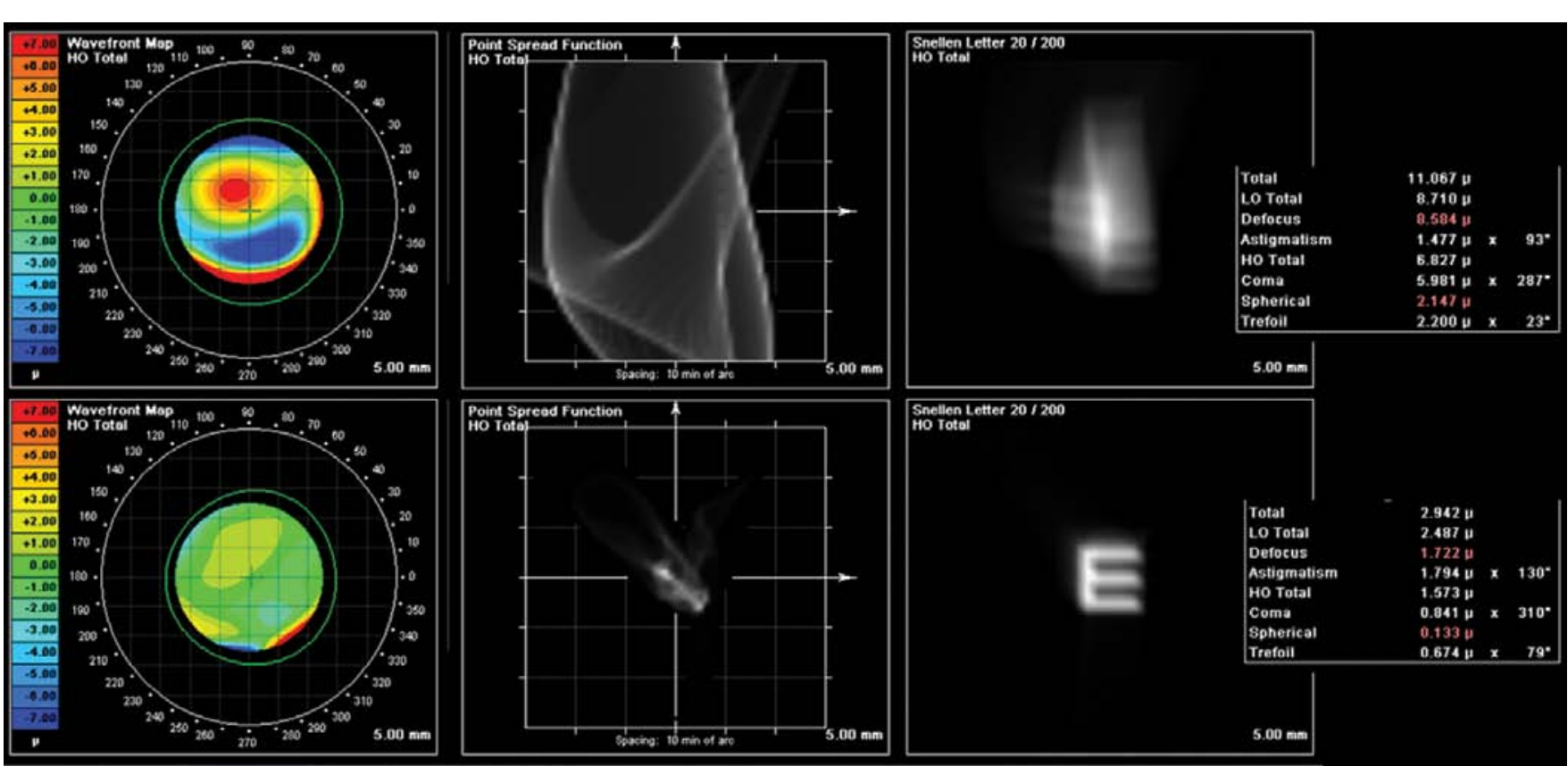

Fig. 2: Ocular aberrometric analysis before and after contact lens fitting. In the top of the figure, the prefitting aberrometric data is displayed whereas the low part of the figure shows the same information but 15 days after contact lens fitting. From left to right, the following information is displayed: higher order aberration (HOA) wavefront map, simulated point spread function (PSF) (the image generated by the ocular optical system of a point source or point object), simulated image of a Snellen E optotype and numerical data of the Zernike terms analyzed

with high astigmatism. This contact lens with a central RGP zone and a soft skirt was associated to some problems, such as the breaking of the transition zone between the RGP zone and the soft skirt or the limitation in the oxygen permeability. Indeed, these authors reported that separation of the lens at the bonded junction and an inability to insert and remove the lens accounted for the majority of failures with this modality of CL. ${ }^{16}$ Recently, new models of hybrid CLs have been developed and are available in the clinical practice. One of these new generation hybrid contact lens is the ClearKone which is designed to be fitted in keratoconus eyes. This lens has a new reverse geometry and is fitted considering the minimal vault needed to clear the cone and the skirt curvature that properly lands inside the limbus and extends onto the sclera. Although the lens was designed to be fitted in keratoconus, creating a central lacrimal lens able to neutralize the corneal irregularity and then providing an excellent visual quality, other options may also possible. We report for the first time a case of ClearKone fitting in a highly astigmatic and steep cornea after PKP in which CL fitting with different types of RPG CLs had previously been unsuccessful and a significant donor-host misalignment was present at the nasal area.

In our case, the tear layer pooled under the reverse geometry zone was able to neutralize corneal irregularity and to minimize HOAs. This was consistent with the subjective patient's perception. She referred a significant improvement of the visual quality with the $\mathrm{CL}$ as well as a comfortable wear. It should be considered that the surrounding of the RGP central zone, containing the corrective optical power and the reverse geometry, by a soft skirt contributes to the centration and stability of the lens in the eye, even in this case with this nasal donor-host misalignment. The RGP zone was of a somewhat smaller diameter than the grafted cornea and therefore the soft skirt covered completely the donor-host transition zone, including the area of misalignment where a bubble was present. This peripheral bubble did not induce corneal staining or any other type of corneal problem at 1 year of contact lens wearing.

Reverse geometry hybrid contact lenses may be a potentially useful option for achieving a complete visual rehabilitation in corneas with high astigmatism and irregularity after PKP, especially when areas of donor-host misalignment are present. This first clinical evidence should be confirmed in future studies including significant samples of cases. Further studies are required to confirm the clinical utility of this lens and long term tolerability.

\section{REFERENCES}

1. Bigar F, Herbort CP. Corneal transplantation. Curr Opin Ophthalmol 1992;3:473-481.

2. Rabinowitz YS. Keratoconus. Surv Ophthalmol 1998;42: 297-319.

3. Mannis MJ, Zadnik K. Refracting the corneal graft. Surv Ophthalmol 1990;34:436-440.

4. Beekhuis WH, Van Rij G, Eggink FA, Vreugdelhil W, Schoevaart CE. Contact lenses following keratoplasty. CLAO J 1991;17: 27-29. 
5. Ozkurt Y, Atakan M, Gencaga T, Akkaya S. Contact lens visual rehabilitation in keratoconus and corneal keratoplasty. J Ophthalmol 2012;832070.

6. Ho SK, Andaya L, Weissman BA. Complexity of contact lens fitting following penetrating keratoplasty. Int Contact Lens Clin 1999;26:163-167.

7. Wietharn BE, Driebe WT Jr. Fitting contact lenses for visual rehabilitation after penetrating keratoplasty. Eye Contact Lens 2004;30:31-33.

8. Szczotka LB, Lindsay RG. Contact lens fitting following corneal graft surgery. Clin Exp Optom 2003;86:244-249.

9. Gruenauer-Kloevekorn C, Kloevekorn-Fischer U, Duncker GI. Contact lenses and special back surface design after penetrating keratoplasty to improve contact lens fit and visual outcome. $\mathrm{Br}$ J Ophthalmol 2005;89:1601-1608.

10. Eggink FA, Nuijts RM. A new technique for rigid gas permeable contact lens fitting following penetrating keratoplasty. Acta Ophthalmol Scand 2001;79:245-250.

11. Gomes JA, Rapuano CJ, Cohen EJ. Topographic stability and safety of contact lens use after penetrating keratoplasty. CLAO J 1996;22:64-69.

12. Genvert GI, Cohen EJ, Arentsen JJ, Laibson PR. Fitting gaspermeable contact lenses after penetrating keratoplasty. Am J Ophthalmol 1985;99:511-514.

13. Katsoulos C, Nick V, Lefteris K, Theodore M. Fitting the postkeratoplasty cornea with hydrogel lenses. CLAO J 2009;32: 22-26.
14. Lagnado R, Rubinstein MP, Maharajan S, Dua HS. Management options for the flat corneal graft. Cont Lens Anterior Eye 2004; 27:27-31

15. Lin JC, Cohen EJ, Rapuano CJ, Laibson PR. RK4 (reversegeometry) contact lens fitting after penetrating keratoplasty. Eye Contact Lens 2003;29:44-47.

16. Binder PS, Kopecky L. Fitting the SoftPerm contact lens after keratoplasty. CLAO J 1992;18:170-172.

\section{ABOUT THE AUTHORS}

\section{Rafael J Pérez-Cambrodí}

Teacher/Clinician, Department of Ophthalmology, Medimar International Hospital, Spain

\section{Pedro Ruiz-Fortes}

Clinician, Department of Ophthalmology, Medimar International Hospital, Spain

\section{David P Piñero Llorens (Corresponding Author)}

Teacher/Clinician, Department of Optics, Pharmacology and Anatomy University of Alicante, Spain, Phone: 34965903500, e-mail: david. pinyero@ua.es 